\title{
Lattice- and molecular-dynamics studies of phase transitions inCsLiSO4
}

\section{Citation}

Katkanant, V., H. M. Lu, and J. R. Hardy. 1992. "Lattice- and Molecular-Dynamics Studies of Phase Transitions inCsLiS04." Physical Review B 46 (10) (September 1): 5982-5988. doi:10.1103/ physrevb.46.5982.

\section{Published Version}

doi:10.1103/PhysRevB.46.5982

\section{Permanent link}

http://nrs.harvard.edu/urn-3:HUL.InstRepos:32430667

\section{Terms of Use}

This article was downloaded from Harvard University's DASH repository, and is made available under the terms and conditions applicable to Other Posted Material, as set forth at http:// nrs.harvard.edu/urn-3:HUL.InstRepos:dash.current.terms-of-use\#LAA

\section{Share Your Story}

The Harvard community has made this article openly available.

Please share how this access benefits you. Submit a story.

Accessibility 


\title{
Lattice- and molecular-dynamics studies of phase transitions in $\mathrm{CsLiSO}_{4}$
}

\author{
V. Katkanant \\ Department of Physics, California State University, Fresno, California 93740-0037
}

\author{
H. M. Lu and J. R. Hardy* \\ Department of Physics and Center for Electro-Optics, University of Nebraska, Lincoln, Nebraska 68588-0111
}

(Received 28 February 1992)

\begin{abstract}
We report results of a simulation of the phase transitions in $\mathrm{CsLiSO}_{4}$. These are based on our previously developed method for calculating parameter-free potential-energy surface for ionic molecular crystals. Our lattice-dynamical and molecular-dynamics studies show that the room-temperature (Pnam) phase is unstable and transforms to the observed low-temperature $\left(P 112_{1} / n\right)$ phase over approximately 200-280 K. The unstable modes of the Pnam phase have maximum instability at the zone center, which indicates a possible phase transformation without a cell multiplication. The rotational ordering of tetrahedral $\mathrm{SO}_{4}{ }^{2-}$ was found to be the driving mechanism of these phase transitions. The quality of the agreement between theoretical and experimental structural parameters and transition temperatures confirms that our potentials for $\mathrm{Li}^{+}$containing sulfates are of comparable accuracy to those for other alkali sulfates.
\end{abstract}

\section{INTRODUCTION}

Cesium lithium sulfate is a potassium selenate family $A_{2} B X_{4}$ compound with two different alkali metal ions in place of the $A_{2}$ ions and an $\mathrm{SO}_{4}^{2-}$ molecular ion as $B X_{4}$. It undergoes two different successive phase transitions below room temperature. ${ }^{1}$ At room temperature, it belongs to the orthorhombic Pnam $\left(D_{2 h}^{16}\right)$ space group with four formula units per unit cell and transforms to a monoclinic $P 112_{1} / n\left(C_{2 h}^{5}\right)$ phase at $202 \mathrm{~K}$. Aleksandrov et $a l .^{2}$ measured the integrated intensity of the $\mathrm{x}$-ray (021) reflection, forbidden in the orthorhombic phase, which measures the monoclinic distortion of the unit cell in the low-temperature phase, and the deviation of the monoclinic angle $(\alpha)$ from $90^{\circ}$, over a wide temperature range below the phase transition. These saturated at about $160 \mathrm{~K}$ for the $\mathrm{x}$-ray intensity and $150 \mathrm{~K}$ for the angle $\alpha$. Hence the authors of Ref. 1 determined the lowtemperature crystal structure at $163 \mathrm{~K}$ and found it to have the same number of atoms per unit cell as at high temperature. There is an intermediate phase at $206 \mathrm{~K}$, observed under a microscope, ${ }^{1}$ showing a pattern of twins which attests to the disappearance of the symmetry elements perpendicular to the $\mathbf{b}$ and $\mathbf{c}$ axis. These twins vanished below $199 \mathrm{~K}$. Furthermore, it was also shown that an abrupt increase in the domain size to a width of $\sim 1 \mathrm{~mm}$ occurs at $200 \mathrm{~K}$. These domain structures undergo reversible changes under the influence of the mechanical stress $T_{12} \cdot{ }^{3}$ This phase, occurring below 202 $\mathrm{K}$, was shown to be a ferroelastic phase transition of the second kind. This transition does not alter the number of atoms in a unit cell. ${ }^{2,3}$

To date, only some properties of these crystals have been extensively investigated experimentally. ${ }^{1-3}$ In this paper, we report theoretical calculations on these compounds for the room-temperature and low-temperature phases. This work will be seen to be related to ideas of Hasebe and Asahi, ${ }^{4}$ who studied mixed crystals of $\mathrm{LiRb}_{x} \mathrm{Cs}_{1-x} \mathrm{SO}_{4}$ and found that the ferroelectric phase transition is completely suppressed at $x=0.95$ as the depths of the double-well potential for the $\mathrm{SO}_{4}^{2-}$ ion became shallower on substitution of $\mathrm{Cs}$ for $\mathrm{Rb}$. In order to understand the mechanism causing these phase transitions, investigations on interionic interactions of these ionic molecular solids were required. These studies were undertaken to verify the validity of our $a b$ initio model of the potentials in ionic molecular crystals in which the electron covalency within the molecular ions substantially affects the interionic interactions. ${ }^{5-7}$ This model leads to a parameter-free description of the potential-energy surfaces for such ionic molecular crystals. We then performed first-principles static structural relaxation, molecular-dynamics simulation, and lattice-dynamics studies for the room-temperature orthorhombic phase and the lower-temperature monoclinic structure of $\mathrm{CsLiSO}_{4}$, and predicted with good accuracy the transition from the former to the latter.

\section{AB INITIO POTENTIAL-ENERGY SURFACES}

To obtain quantitative knowledge and to understand the physics of these phase transitions in $\mathrm{CsLiSO}_{4}$, an accurate theoretical potential-energy surface involving both inter- and intramolecular interactions is needed. We first calculate the charge density of the whole $\mathrm{SO}_{4}^{2-}$ molecular ions using a quantum-chemistry structural optimization. This is achieved by searching for the atomic configuration that gives the lowest self-consistent Hartree-Fock energy. A full description of the procedure for obtaining both intra- and interionic potentials from 
these quantum-chemistry calculations has been given elsewhere. ${ }^{5-7}$ Only a brief discussion related to $\mathrm{CsLiSO}_{4}$ will be presented here. The calculated optimized structures gave near-perfect tetrahedra with average $\mathrm{S}-\mathrm{O}$ bond lengths of $1.472 \AA($ Pnam $)$ and $1.475 \AA\left(P 112_{1} / n\right)$ compared to the experimental values of 1.457 and $1.468 \AA$ for the room- and low-temperature structures, respectively. Owing to the covalency within the molecular ion, a Gordon-Kim model with the pairwise interactions ${ }^{8}$ is inadequate to describe the intramolecular interactions, thus a harmonic expansion of the energy of the free $\mathrm{SO}_{4}^{2-}$ ion was constructed using the force constants (or the second derivatives of the total energy of the $\mathrm{SO}_{4}^{2-}$ ion with respect to distortions from this optimized structure). This provides an excellent description of the intramolecular interactions between $\mathrm{S}-\mathrm{O}$ and $\mathrm{O}-\mathrm{O}$ atoms within the same $\mathrm{SO}_{4}^{2-}$ ion, as will be seen later from the good agreement between the experimental and theoretical calculations. On the other hand, the "rigid-ion" assumption using Gordon-Kim technique is valid for the intermolecular interactions, due to the purely ionic bonding. From the optimized structure of the whole $\mathrm{SO}_{4}^{2-}$ ion, one can also obtain charge densities for the individual $\mathrm{S}$ and $\mathrm{O}$ atoms in the spirit of Mulliken population analysis. ${ }^{9}$ These calculated charge distributions of the atoms, from this Mulliken population process, may not have the spherical behavior required by the Gordon-Kim model. To overcome this, a modification of the $p$ or $d$ orbitals using only the radial part wave functions of these orbitals was employed. For Cs ${ }^{+}$and $\mathrm{Li}^{+}$we simply use the closed-shell charged distribution for the free ions. With these spherical charge-density distributions and the Gordon-Kim electron-gas model, we then computed the short-range pair potentials between these ions. The long-range Coulomb interactions were also calculated using the fractional ionicities of the $\mathrm{S}$ and $\mathrm{O}$ atoms, as +1.6371 for $\mathrm{S}$ and -0.9093 for $O$ from Mulliken population analysis. Of course, the ionicity charge for $\mathrm{Cs}$ and $\mathrm{Li}$, closed-shell configurations, is +1 .

\section{STATIC RELAXATION CALCULATIONS OF $\mathrm{CsLiSO}_{4}$ STRUCTURES}

After obtaining the intramolecular and intermolecular interaction potentials for both Pnam $\left(D_{2 h}^{16}\right)$ and $P 112_{1} / n$ structures, we performed static relaxation calculations for $\mathrm{CsLiSO}_{4}$ with and without crystal structure symmetry constraints. We found that our $a b$ initio potentials are very satisfactory, since the results showed that the theoretical structures with zero forces on the basis ions and zero stresses agree very well with the experimental data for both high- and low-temperature phases. Moreover, both the relaxed structures which were subject to the constraints of Pnam and $P 112_{1} / n$ symmetries (i.e., only the structural atomic positions and the lengths of the lattice constants were allowed to vary) and the general relaxation without the symmetry constraints gave identical structures. The minimum energies for the highand low-temperature structures are -21.8581 and $-21.9033 \mathrm{eV} /$ formula unit, respectively. The parameters for the relaxed structures along with the experimen- tal values for the Pnam and $P 112_{1} / n$ structures are given in Tables I and II. Figures 1 and 2 show comparisons of the projections of the experimental and theoretical positions parallel to each of the three orthorhombic axes $\mathbf{a}, \mathbf{b}$, and $\mathrm{c}$ for the Pnam and $P 112_{1} / n$ structures.

As can be seen from Figs. 1 and 2 and from the prototypic parameters given in Table $I$ and II, our relaxed structures agree very well with experiment, particularly as far as the reduced (fractional) basis vectors are concerned. These tables show not only the lattice constants and atomic positions but also the comparisons, listed in both percent differences and in angstroms. The largest difference is only about $0.10-0.15 \AA$ for the $x$ coordinate of $\mathrm{Li}$ and $x$ coordinate of $\mathrm{O}(2)$ for both structures. This discrepancy is comparable with the thermal fluctuation of the atomic positions at both temperatures. The lattice constants for the relaxed structures are overall shorter than the experimental values, which is a rather general
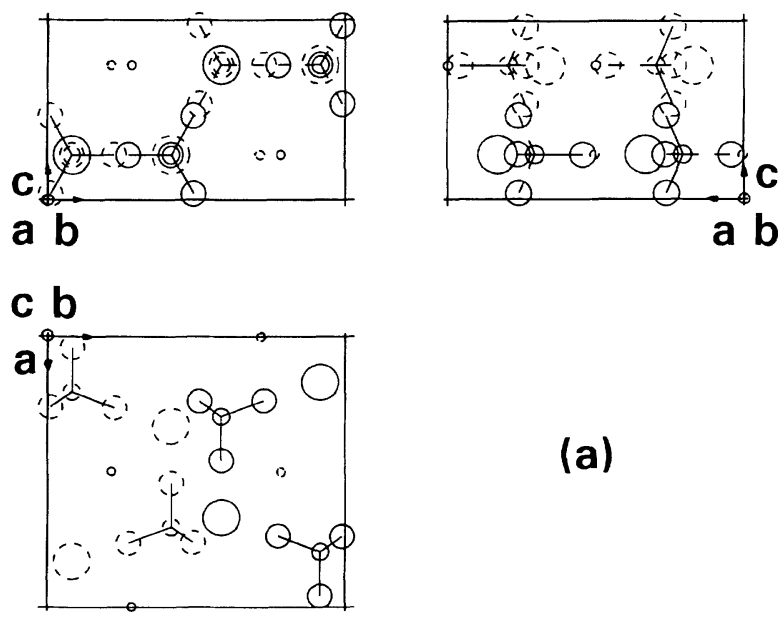

a b

(a)
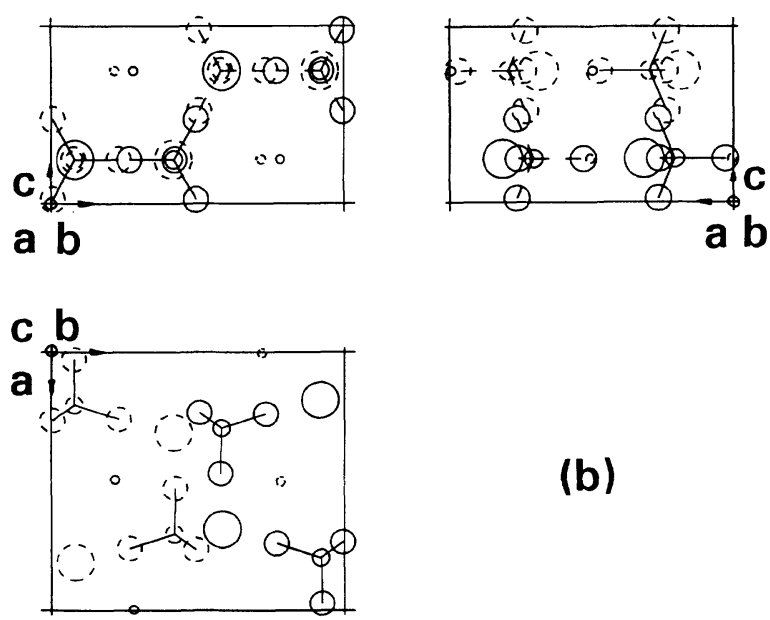

(b)

FIG. 1. Projections of the (a) experimental and (b) theoretical static structure of $\mathrm{CsLiSO}_{4}$ in the Pnam phase. A lattice vector pointing out of the page is represented by a circle. The smallest circles represent the Cs atoms and the largest circles represent $\mathrm{Li}$ atoms. Bonds (straight lines) connect each $S$ and the nearest four $\mathrm{O}$ atoms. 
TABLE I. Experimental (Ref. 1) and theoretical structural parameters for the Pnam structure of $\mathrm{CsLiSO}_{4}$. (The lattice constants are given in angstroms.)

\begin{tabular}{|c|c|c|c|c|}
\hline Parameters & Experiment & Theory & $\%$ Diff. & Diff. \\
\hline$a$ & 8.820 & 8.360 & 5 & 0.46 \\
\hline$b$ & 9.456 & 9.223 & 2 & 0.23 \\
\hline$c$ & 5.456 & 5.306 & 3 & 0.15 \\
\hline$x / a$ of $\mathrm{S}(1)$ & 0.2040 & 0.2049 & 0 & -0.01 \\
\hline$y / b$ of $S(1)$ & 0.0831 & 0.0791 & 5 & 0.04 \\
\hline$x / a$ of $\operatorname{CS}(1)$ & 0.9985 & 0.9951 & 0 & 0.03 \\
\hline$y / b$ of $\operatorname{Cs}(1)$ & 0.2843 & 0.2820 & 1 & 0.02 \\
\hline$x / a$ of $\operatorname{Li}(1)$ & 0.3310 & 0.3142 & 5 & 0.15 \\
\hline$y / b$ of $\operatorname{Li}(1)$ & 0.4160 & 0.4161 & 0 & 0.00 \\
\hline$x / a$ of $\mathrm{O}(1)$ & 0.2632 & 0.2616 & 1 & 0.01 \\
\hline$x / b$ of $\mathrm{O}(1)$ & 0.2260 & 0.2301 & -2 & -0.04 \\
\hline$x / a$ of $\mathrm{O}(2)$ & 0.0412 & 0.0289 & 30 & 0.11 \\
\hline$y / b$ of $\mathrm{O}(2)$ & 0.0840 & 0.0778 & 7 & 0.06 \\
\hline$x / a$ of $\mathrm{O}(3)$ & 0.2609 & 0.2645 & -1 & -0.03 \\
\hline$y / b$ of $\mathrm{O}(3)$ & 0.0101 & 0.0041 & 59 & 0.06 \\
\hline$z / c$ of $\mathrm{O}(3)$ & 0.0325 & 0.0235 & 28 & 0.05 \\
\hline
\end{tabular}
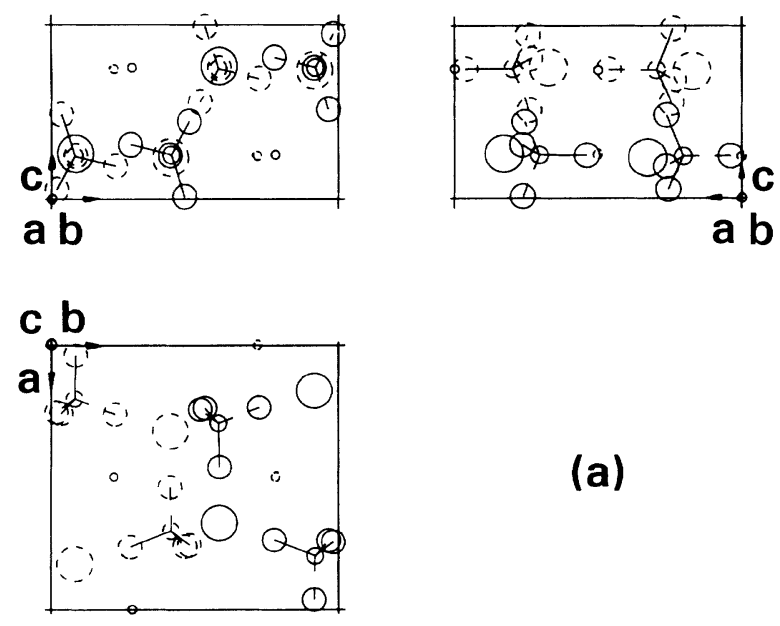

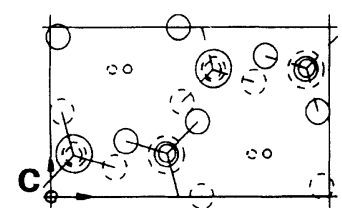

a b

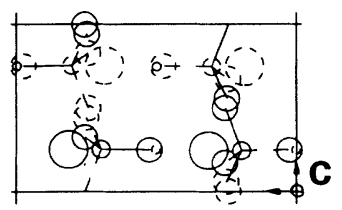

a b

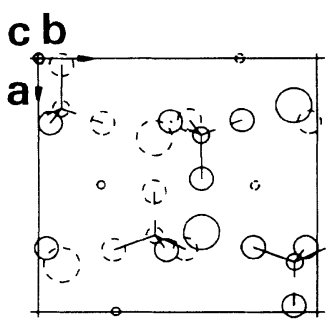

(b)

FIG. 2. Projections of the (a) experimental and (b) theoretical static structure of $\mathrm{CsLiSO}_{4}$ in the $P 112_{1} / n$ phase. feature for large unit cell simulations using Gordon-Kim potentials. The maximum discrepancy is $5 \%$ for the $a$ axis of the Pnam structure. However, the shortenings seem rather random for the three axes for both structures, but the ratios of $b / c$ are 1.738 and 1.722 close to the experimental values 1.733 and 1.729 of Pnam and $P 112_{1} / n$ structures, respectively. It should be pointed out that these $\mathbf{b}$ and $\mathbf{c}$ axes form the monoclinic angle $\alpha$ in the low-temperature phase. This indicates that, while the discrepancies in the absolute lengths of the axes are significant, the near uniformly contracted theoretical structure should still preserve those basic symmetries of the system relevant to the phase transitions under study.

From these tables, it can be seen that in order to specify the two structures requires, respectively, 16 and 24 parameters for the Pnam and $P 112_{1} / n$ structures, each of which depends explicitly on the accuracy of our potentials. The fact that almost all of these parameters are reproduced to within a few percent (the few larger values relate to components whose actual values are an order of magnitude smaller than the others) by potentials that are entirely parameter-free argues strongly both for a high degree of reliability in these potentials and of the underlying theory. Overall the comparison of the lattice constants and the positions of the ions in these structures with the positions determined by experiment provides a sensitive test of the validity of the theoretical potentialenergy surface. This excellent agreement lead us to conclude that we have indeed found a realistic potentialenergy model for describing the sulfate ionic molecular solids.

TABLE II. Experimental (Ref. 1) and theoretical structural parameters for the $P 112_{1} / n$ low-temperature structure of $\mathrm{CsLiSO}_{4}$. (The lattice constants are given in angstroms.)

\begin{tabular}{lclrr}
\hline Parameters & Experiment & Theory & \% Diff. & Diff. \\
\hline$a$ & 8.834 & 8.541 & 3 & 0.29 \\
$b$ & 9.379 & 9.028 & 4 & 0.35 \\
$c$ & 5.423 & 5.243 & 3 & 0.18 \\
$x / a$ of $\mathrm{S}(1)$ & 0.2030 & 0.1975 & 3 & 0.05 \\
$y / b$ of $\mathrm{S}(1)$ & 0.0818 & 0.0823 & -1 & 0.00 \\
$z / c$ of $\mathrm{S}(1)$ & 0.2470 & 0.2457 & 1 & 0.01 \\
$x / a$ of $\mathrm{Cs}(1)$ & 0.9992 & 0.9982 & 0 & 0.01 \\
$y / b$ of $\mathrm{Cs}(1)$ & 0.2811 & 0.2768 & 2 & 0.04 \\
$z / c$ of $\mathrm{Cs}(1)$ & 0.7538 & 0.7516 & 0 & 0.01 \\
$x / a$ of $\mathrm{Li}(1)$ & 0.3270 & 0.3144 & 4 & 0.11 \\
$y / b$ of $\mathrm{Li}(1)$ & 0.4160 & 0.4155 & 0 & 0.00 \\
$z / c$ of $\mathrm{Li}(1)$ & 0.2380 & 0.2457 & -3 & -0.04 \\
$x / a$ of $\mathrm{O}(1)$ & 0.2632 & 0.2556 & 3 & 0.07 \\
$y / b$ of $\mathrm{O}(1)$ & 0.2239 & 0.2303 & -3 & -0.06 \\
$z / c$ of $\mathrm{O}(1)$ & 0.1886 & 0.1729 & 8 & 0.09 \\
$x / a$ of $\mathrm{O}(2)$ & 0.0379 & 0.0251 & 34 & 0.11 \\
$y / b$ of $\mathrm{O}(2)$ & 0.0851 & 0.0850 & 0 & 0.00 \\
$z / c$ of $\mathrm{O}(2)$ & 0.2491 & 0.2501 & 0 & -0.01 \\
$x / a$ of $\mathrm{O}(3)$ & 0.2555 & 0.2516 & 2 & 0.03 \\
$y / b$ of $\mathrm{O}(3)$ & 0.0191 & 0.0276 & -45 & -0.08 \\
$z / c$ of $\mathrm{O}(3)$ & 0.0552 & 0.0577 & -5 & -0.01 \\
$x / a$ of $\mathrm{O}(4)$ & 0.2612 & 0.256 & 2 & 0.05 \\
$y / b$ of $\mathrm{O}(4)$ & 0.0348 & 0.0418 & -20 & -0.07 \\
$z / c$ of $\mathrm{O}(4)$ & 0.4867 & 0.5014 & -3 & -0.08 \\
\hline \hline
\end{tabular}




\section{LATTICE-DYNAMIC CALCULATIONS $\mathrm{OF} \mathrm{CsLiSO}_{4} \mathrm{STRUCTURES}$}

To examine the nature of lattice vibrations, phonon dispersion curves along the $\mathbf{a}^{*}, \mathbf{b}^{*}$, and $\mathbf{c}^{*}$ have been obtained by lattice-dynamic calculations for both Pnam and $P 112_{1} / n$ structures. Furthermore, we want to investigate the possible instabilities allowed by the potential-energy surface for these configurations of the $\mathrm{CsLiSO}_{4}$ system. Moreover, since experimentally these two structures have the same number of atoms per unit cell, we wish to know if there is any indication of cell multiplication shown by the unstable modes.

We performed our lattice-dynamic calculation for the relaxed theoretical structures along [100], [010], and [001] directions. The dispersion curves, angular frequency $\left(\omega^{2} /|\omega|\right)$ versus wave vector $(\mathbf{q})$, along [100] are shown in Figs. 3(a) and 3(b) for the Pnam and P $112_{1} / n$ structures, respectively. There are three acoustic modes,
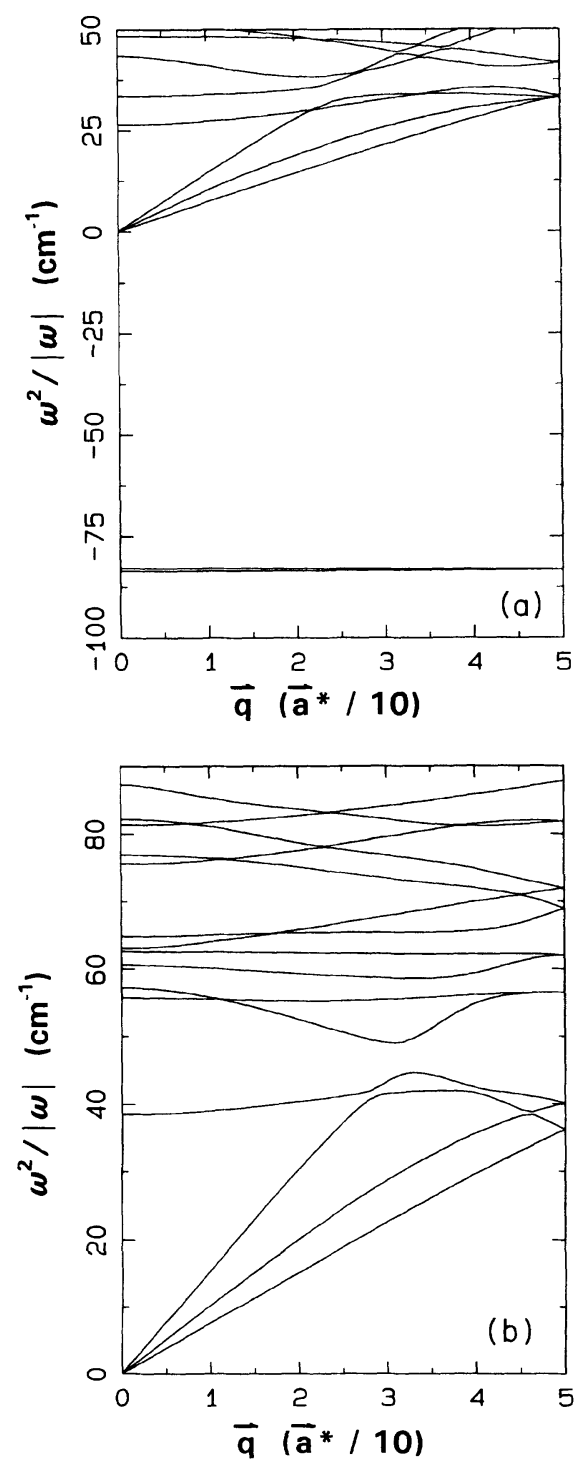

FIG. 3. Dispersion curves along the [100] direction for the theoretical (a) Pnam and (b) $P 112_{1} / n$ structures. as expected, starting from zero wave vector in both phases. However, in the Pnam phase, there are two negative branches, which represent imaginary frequencies, along all three directions. The appearance of these negative branches indicates that the theoretical Pnam structure is actually unstable, while the $P 112_{1} / n$ phase is stable down to zero temperature against the moleculardynamics (MD) relaxations which we are going to discuss in the following section. This is confirmed by our not finding, for the latter, any imaginary unstable branches from lattice-dynamic calculations. We found steep minima of the negative branches (point of maximum instability) along [010] and [001] to occur at the zone center. On the other hand, along the [100] direction these unstable branches are almost flat. However, the absolute minimum of the lowest branch still can be identified as lying at the zone center. Thus as far as the symmetry of the instability is concerned, our lattice-dynamic calculation agrees well with experiment and complements our MD finding that there is no cell-multiplying transition. Thus the number of atoms in the unit cells of both phases is not modified by the phase transition.

It is worth mentioning that the instability shown by the Pnam structure indicates that this structure is actually unstable. This is surprising since the Pnam relaxed structure calculated by our static relaxation revealed that all forces on the ions and the stresses were zero. The probable explanation is that the Pnam structure is not at an absolute minimum but is at a fairly broad saddle point on the potential-energy surface. These relaxations give the crystal structures that are at extrema of the theoretical potential-energy surface.

\section{MOLECULAR-DYNAMIC SIMULATIONS OF THE PHASE TRANSITION}

Initially, when we succeeded in reproducing the static Pnam structure of the room-temperature $\mathrm{CsLiSO}_{4}$, we then performed molecular-dynamics calculations to simulate and examine directly the lower-temperature phase structure. Our molecular-dynamics simulations follow a constant-pressure algorithm. ${ }^{10}$ Periodic boundary conditions are introduced in order for the lattice being simulated to behave as an infinite system. No extra restriction was applied, in order to ensure that the simulations were as realistic as possible and would provide a visual representation of the movements of the ions. We quenched the sample being simulated, which consisted of one relaxed Pnam unit cell (28 ions), by gradually reducing the total kinetic energy of the sample in stages to zero, to reach the ground state. We found that we could indeed reproduce realistically the experimentally observed monoclinic $P 112_{1} / n$ structure at low temperature, the angle $\alpha$ having a value of $89.767^{\circ}$ compared with the experimental value $89.75^{\circ}$ (or $89^{\circ} 45^{\prime}$ ). ${ }^{1}$ This shows excellent agreement while the other angles $\beta$ and $\gamma$ are very close to $90^{\circ}$, i.e., $89.968^{\circ}$ and $89.976^{\circ}$. Next we performed the static relaxation for the $P 112_{1} / n$ structure and the results are shown in Table II and Fig. 2 as mentioned in the preceding section. Figures $4(\mathrm{a})$ and $4(\mathrm{~b})$ show the structures and atomic positions of the MD simulation at 300 and $0 \mathrm{~K}$, respectively. In these figures, the average $\mathrm{S}-\mathrm{O}$ bonds are indi- 
cated by straight lines. Clearly the structure in Fig. 5(a) is virtually the same as experiment and the statically relaxed $P 112_{1} / n$ structure of Fig. 2 . The unit cell is monoclinic with the average lattice constants $a=8.535 \AA$, $b=9.032 \AA$, and $c=5.244 \AA$. These values are shorter than the experimental values by $3 \%, 4 \%$, and $3 \%$, discrepancies of the same order as for the relaxed $P 112_{1} / n$ structures, and the ratio of $b / c$ is 1.722 .

We also performed a MD quench of a larger supercell sample. It consisted of eight of the Pnam unit cells of $\mathrm{CsLiSO}_{4}$, in total 224 ions. The supercell was obtained by doubling in $\mathbf{a}, \mathbf{b}$, and $\mathbf{c}$ directions to lessen artificial correlations in all three directions. The results are shown in Fig. 5(a) and compared with the single cell simulations as shown in Fig. 5(b) but multiplied to $2 a \times 2 b \times 2 c$. As can be seen, the $\mathrm{SO}_{4}^{2-}$ on the same $b c$ plane of the Pnam structure align along the mirror plane parallel to $\mathbf{b}$. In the low-temperature structure the $\mathrm{SO}_{4}^{2-}$ on the same $b c$ plane of the single-cell rotate as a whole about the a axis by $14^{\circ}$ from the mirror plane. For the double cell, it is obvious from the figure that there are two equally probable positions for the $\mathrm{SO}_{4}^{2-}$ to occupy, rotated by $\pm 14^{\circ}$ in close agreement with the experimental findings of Ref. 1 . Hence we can conclude that the phase transitions in ferroelastic $\mathrm{CsLiSO}_{4}$ compounds are associated with the ordering of the $\mathrm{SO}_{4}^{2-}$ tetrahedra in one or other well. Our MD simulations indicated that for both the single- and double-cell simulations the energies of these two structures are very close: -21.9037 and -21.9029

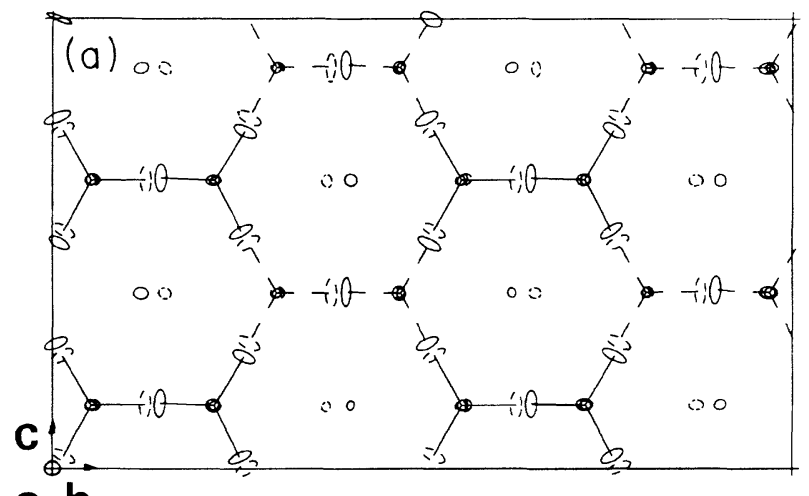

a b

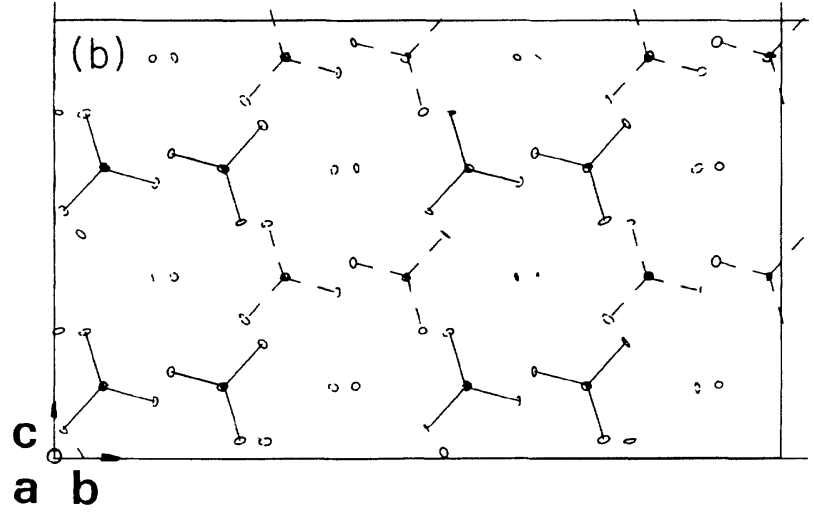

FIG. 4. bc cross sections of the MD average structures of $\mathrm{CsLiSO}_{4}$ at $(\mathrm{a}) \sim 300 \mathrm{~K}$ and $(\mathrm{b}) \sim 0 \mathrm{~K}$.
$\mathrm{eV} /$ formula unit. The single cell has a lower energy by only $0.76 \mathrm{meV} /$ formula unit (corresponding to a temperature of $9 \mathrm{~K}$ ). The fact that this energy difference is a lot less than $200 \mathrm{~K}$ may explain the sluggish initiation of the transformation between 206 and 202 K, evidenced by slow twin nucleation.

We also performed MD simulations starting from the low-temperature, $P 112_{1} / n$, structure. Before these MD simulations on this structure, we desired to remove any residual excess energy from the lattice that may have been left over from the static relaxation and thus to assure ourselves that the system was truly in its ground state. The sample was "quenched" to $0 \mathrm{~K}$ so that there was no kinetic energy left in the lattice and it had reached the state of minimum static energy. We then ran MD simulations on this quenched ground state to study the dynamical behavior of these systems as they are "heated" in the computer, by the slow input of additional kinetic energy. In addition to revealing global phase transformations, this will also reveal the onset of local disorder (probably dynamical), and transitions between static and dynamic disorder. Of particular importance is disorder closely associated with an overall structural transformation. In the first run, thermal energy
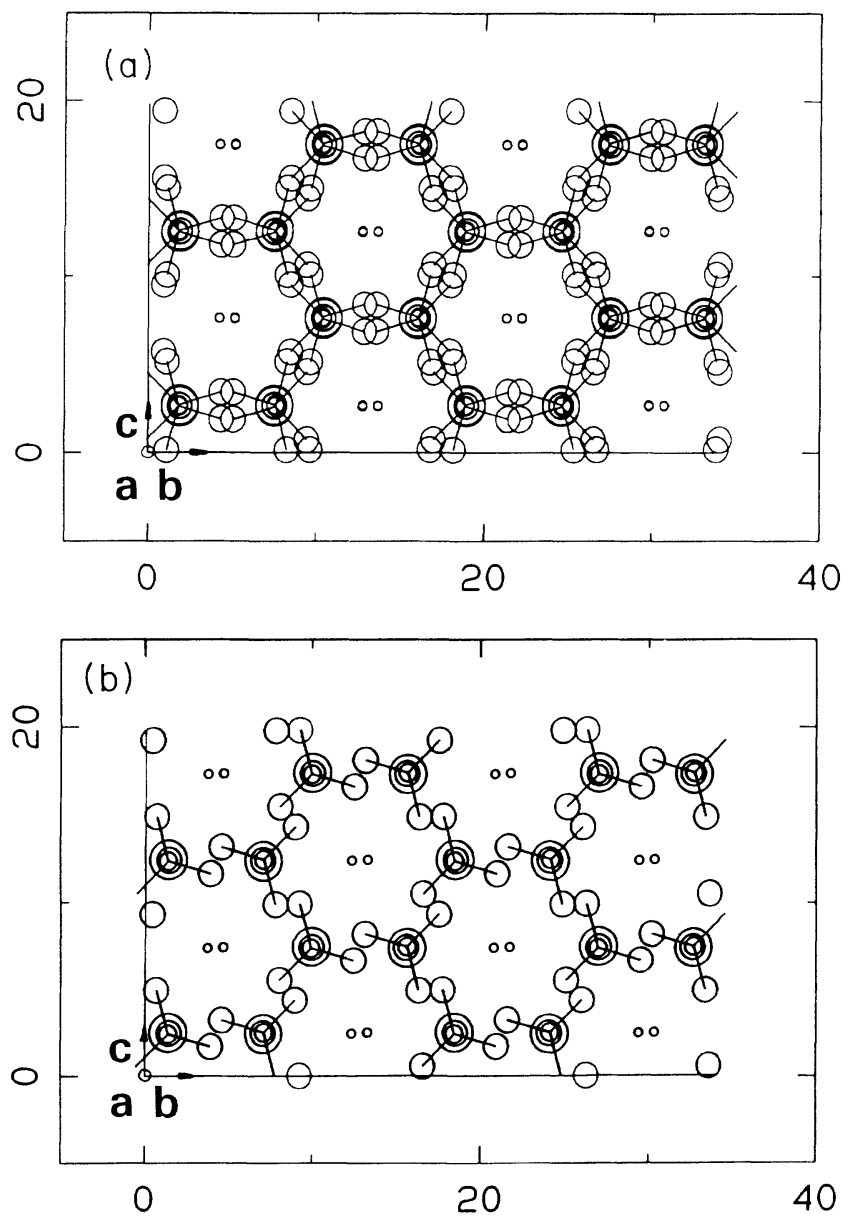

FIG. 5. bc cross sections for the $2 a \times 2 b \times 2 c$ supercell structure of $\mathrm{CsLiSO}_{4}$ obtained by (a) molecular-dynamics relaxation to $T=0 \mathrm{~K}$ and (b) static relaxation. 
equivalent to $20 \mathrm{~K}$ was added to the lattice after successive time intervals. The time intervals, during which the program collected data for the average velocities, positions, etc. were chosen to be $12 \mathrm{ps}$ long. This was long enough to ensure genuine thermalization for each step before data are collected and averaged. We ran the simulation from 0 to $400 \mathrm{~K}$, at each temperature the average ionic positions and their thermal ellipsoids were saved, as well as the average cell side lengths, lattice vector angles, cell volume, and total kinetic and potential energies. To verify the results of this run, a second one was done with the same parameters, except that the time intervals were doubled to $24 \mathrm{ps}$. There are no significant differences between these two runs.

In order to find the transition temperature of these compounds, we first examined the molecular dynamics of a $1 \mathbf{a} \times 2 \mathbf{b} \times 2 \mathbf{c}$ supercell. It can be seen that, on cooling, the system transforms from the high-temperature structure to the low-temperature structure starting at about $250 \mathrm{~K}$ and completing at about $200 \mathrm{~K}$. Clearly, while somewhat higher, this is in very reasonable agreement with the observed onset at about $202 \mathrm{~K}$ and completion at about $160 \mathrm{~K} \cdot{ }^{1-3}$ However, if we return to the dispersion curves in Fig. 3, it can be seen that the unstable branch is very flat, specifically, the minimum at $q=0$ is only $0.5 \mathrm{~cm}^{-1}$ below the zone-boundary (maximum) value. This clearly implies very weak intersulfate coupling and thus considerable difficulty in finding the true ground state in MD simulations with multiple unit cells along a. To verify this we carried out simulations with up to five cells along a, i.e., $5 \mathbf{a} \times \mathbf{b} \times \mathbf{c}$. In every case the system, while transforming over sensibly the same temperature range, assumed the maximum possible periodicity by various types of faulting. Clearly, this represents a basic difficulty in reaching the true ground state, since even though every configuration we examined had a higher static energy, the differences were only $\sim 5-10 \mathrm{~K}$ per formula unit. A similar difficulty is also present for the real system. Apparently, between 202 and $160 \mathrm{~K}$, the structure is very disordered (twins, incommensurations, etc.), indicating that at about $200 \mathrm{~K}$ the guiding potential-energy surface indeed has many closely spaced branches which permit of large disorder (entropy) at negligible cost in energy. Molecular dynamics is ill fitted to reproducing the real behavior in this situation owing to finite supercell size which imposes a maximum cutoff on the scale of allowed fluctuations in both space and time which is dictated limits of the computer. This probably tends to "lock" the system into the state of maximum periodicity since there are then no longer-period fluctuations to which it can relax. Specifically, since the marginally higher energies of the faulted structures are associated with a reduction in the monoclinic shear, it would appear that the elastic component of the free energy plays a major role in the final ordering. In molecular dynamics, this component is largely absent due to the finite wavelength and time cutoffs. As a consequence of this, we would argue that our single-cell (along a) simulation gives us the best estimate of $T_{c}$ for our potentials and the agreement with experiment is such as to support clearly the accuracy of our potentials.

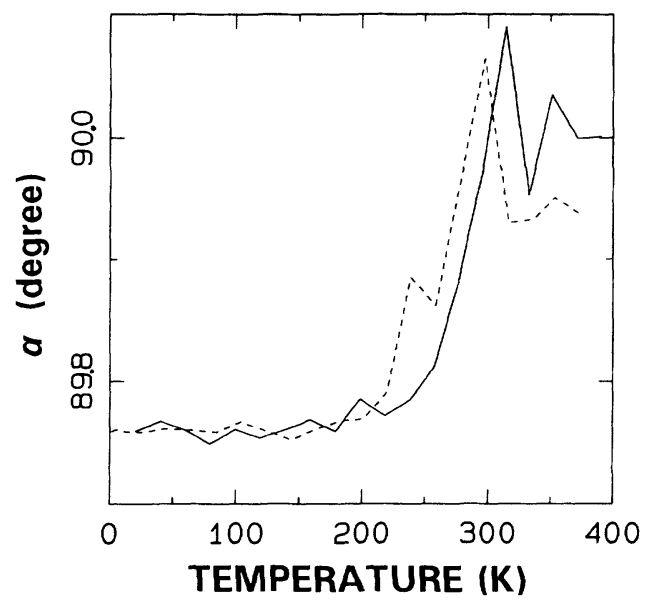

FIG. 6. The order parameter $\alpha$ (the angle between the lattice vectors $b$ and c) as functions of temperature obtained from molecular-dynamics "heating" (solid line) and "quenching" (dashed line).

Finally, in order to obtain a definitive value of $T_{c}$, we display in Fig. 6 the order parameter $\alpha$ (the bc angle) versus temperature for both cooling and heating runs, taken from molecular dynamics simulations starting respectively from Pnam and $P 112_{1} / n$ structures. From these plots, it appears that, on cooling, the transition initiates at about $270 \pm 20 \mathrm{~K}$ and completes by $200 \pm 20 \mathrm{~K}$. On the other hand, on heating, the transformation begins at $230 \pm 20 \mathrm{~K}$ and ends at $290 \pm 20 \mathrm{~K}$. Most of the differences are probably due to finite specimen size. Thus allowing for these uncertainties, we estimate that $T_{c}=280 \pm 20 \mathrm{~K}$ and the transition saturates at approximately $200 \mathrm{~K}$.

\section{DISCUSSION}

In this paper we have described the application of our technique for developing parameter-free interionic potentials for ionic molecular crystals to another isomorph of the $A_{2} B X_{4}$ family of solids, of which we have used $\mathrm{K}_{2} \mathrm{SeO}_{4}$ (Refs. 5 and 6 ) as the prototype member. The present system is qualitatively different in two respects: it contains two dissimilar cations, and one of these is $\mathrm{Li}^{+}$. The former difference is self-evident, the latter is not so apparent. It stems from the fact that $\mathrm{Li}^{+}$has only two electrons and would appear to constitute the worst possible case for use of the Gordon-Kim approach to constructing intra- and interionic potentials. However, our findings for this system are of comparable accuracy to those we have reported for other systems. Our predicted structures agree closely with experiment both for the room-temperature and low-temperature phases. Our prediction of the phase-transition temperature $T_{c}(200-280$ $\mathrm{K}$ versus the experimental value of $160-202 \mathrm{~K}$ ) is percentage wise somewhat less accurate than before, but this discrepancy may well reflect quantum effects in the dynamics of $\mathrm{Li}^{+}$ion: e.g., zero-point motion would tend to destabilize the low-temperature phase. To counter this tendency the $T_{c}$ predicted by classical molecular dynam- 
ics would have to be higher than the observed $T_{c}$, as is the case. Together, these findings provide strong evidence that the Gordon-Kim method performs as well for two electron $\mathrm{Li}^{+}$ions as it does for other alkali ions, and results for $\mathrm{Li}^{+}$based ionic molecular solids should be as reliable as those reported for other compounds.

In conclusion, we would note that once again we have identified the basic driving mechanism of a structural transition as the bistable motion of molecular ionic units $\left(\mathrm{SO}_{4}^{2-}\right)$ in double wells in the crystal potential-energy hypersurface: these wells being present at temperatures far above $T_{c}$. This is entirely in accord with the picture proposed by Kruglik et al. ${ }^{1}$ on the basis of their $\mathrm{x}$-ray structural data. The reconstruction we predicted below $T_{c}$ agrees both qualitatively and quantitatively with that described at the conclusion of their paper.

\section{ACKNOWLEDGMENTS}

The work of V.K. was supported by the Research Corporation. The work of H. M. Lu and J. R. Hardy was supported by the Army Research Office.

[Sov. Phys. Solid State 23, 1426 (1981)].

*Author to whom all correspondence should be addressed.

${ }^{1}$ A. I. Kruglik, M. A. Simonov, E. P. Zhelezin, and Academician N. V. Belov, Dokl. Akad. Nauk SSSR 247, 1384 (1979) [Sov. Phys. Dokl. 24, 596 (1979)].

${ }^{2}$ K. S. Aleksandrov, L. I. Zherebtsova, I. M. Iskornev, A. I. Kruglik, O. V. Rozanov, and I. N. Flerov, Fiz. Tverd. Tela (Leningrad) 22, 3673 (1980) [Sov. Phys. Solid State 22, 2150 (1980)].

${ }^{3}$ K. S. Aleksandrov, M. P. Zaitseva, L. A. Shabanova, and O. V. Shimanskaya, Fiz. Tverd. Tela (Leningrad) 23, 2440 (1981)
${ }^{4}$ K. Hasebe and T. Asahi, Ferroelectrics 96, 63 (1989).

${ }^{5}$ H. M. Lu and J. R. Hardy, Phys. Rev. Lett. 64, 661 (1990).

${ }^{6}$ H. M. Lu and J. R. Hardy, Phys. Rev. B 42, 8339 (1990).

${ }^{7}$ D. Liu, H. M. Lu, F. G. Ullman, and J. R. Hardy, Phys. Rev. B 43, 6202 (1991).

${ }^{8}$ R. G. Gordon and Y. S. Kim, J. Chem. Phys. 56, 3122 (1971).

${ }^{9}$ R. S. Mulliken, J. Chem. Phys. 23, 1833 (1955).

${ }^{10}$ M. Parrinello and A. Rahman, Phys. Rev. Lett. 45, 1196 (1980). 